\title{
Jahrestagung der DGP in Leipzig
}

\section{Phlebologie - national und international}

Die 62. Jahrestagung unserer Gesellschaft findet in diesem Jahr vom 2.-5. September in Leipzig unter dem Tagungsmotto „Phlebologie - national und international“ statt.

Schwerpunkte der Tagung werden die Präsentation des wissenschaftlichen Fortschritts und aktuelle Innovationen im nationalen und internationalen Bereich, die Versorgungssituation in der Praxis und die Fortbildung sowohl für den niedergelassenen Arzt als auch für den jungen Phlebologen sein. Das Kurssystem (Crashkurs Phlebologie, Update Phlebologie, zahlreiche Hands-on-Kurse) behalten wir bei. Speziell für den phlebologischen Nachwuchs bieten wir nach dem Crashkurs am Mittwoch ein PhleboStartUp an, das sich speziell an den studentischen Nachwuchs richtet. Diejenigen, die Lust auf mehr haben, können dann am Donnerstagvormittag das Weiterbildungsforum speziell für junge Phlebologen und solche, die es werden wollen, besuchen.

Zum ersten Mal wird am Freitag und Samstag ein Vortragsstrang Deutsch/Englisch mit Simultanübersetzung angeboten, um der steigenden Nachfrage der Kollegen aus dem angrenzenden Ausland nachzukommen und um uns zu ermöglichen, Einblicke in die Situation der Nachbarländer zu erhalten. Diese Initiative wurde von unseren Kollegen sehr konstruktiv aufgenommen, wir dürfen uns auf ein hervorragendes Expertengremium aus vielen Ländern und zu vielen phlebologischen Inhalten freuen.

Darüber hinaus findet ebenfalls als Novum am Donnerstag eine ganztägige, englischsprachige Ultrasound Master Class statt. Der eintägige Ultraschall Kurs wird von Frau Dr. Mendoza durchgeführt und wendet sich an Einsteiger und Anwender des Ultraschalls in der Phlebologischen Praxis. Er vermittelt einen Überblick über die Diagnostik mit den Schwerpunkten Physiologie des Venensystems, Fluss und Reflux, Untersuchung der Krosse der V. saphena magna, der Stammvenen im Verlauf, der Perforansvenen, Ableitung der dem Patienten angepassten Therapie-Indikation je nach Schallbefund. Die Theorie wird mit vielen LifeDemonstrationen an Patienten ergänzt.

Weiter werden in zahlreichen Hands-onKursen praxisrelevante Therapieformen von erfahrenen Phlebologen gezeigt und von den Teilnehmern selbst geübt.

Eine Anmeldung zum Kongress ist möglich unter: https://phlebologie-2020.de/anmel dung/.

Auch gibt es bereits die Möglichkeit zur Einreichung Ihrer Abstracts unter: https://phlebologie-2020.de/abstracts/.

Darüber hinaus werden neben den klassischen phlebologischen Themen des interdisziplinär besetzten Fachbereichs wie Kompression, Sklerosierung, endovenöse Verfahren und Operationen auch die Wundversorgung, Antikoagulation, Ernährung und moderne Interventionen diskutiert.

Der intensive Dialog der Teilnehmer aus allen Fachbereichen und Berufsgruppen der Phlebologie und ihrer Nachbardisziplinen ist uns ein Anliegen. Nur die enge Vernetzung der beteiligten Fachbereiche ermöglicht eine optimale Patientenversorgung. Gemeinsame Sitzungen mit DGA, DGG, DGL, sowie DGFW/ICW sind geplant, insbesondere ein Rezertifizierungskurs für ICW Wundexperten mit 8 ICW Punkten am Freitag, den 4.9.2020 Am Samstag, den 5.9.2020 findet ein Hausärztetag an unse- 
rem Kongress statt, der die Schnittstellen zwischen Allgemeinmedizin und Phlebologie herausarbeitet. Unter Kooperation mit der DeGUM wird ein Workshop zur Arteriellen Duplex-Sonographie angeboten.

Auch in diesem Jahr bietet es sich für den wissenschaftlichen Nachwuchs an, sich um einen der Wissenschaftspreise zu bewerben, die dankenswerterweise wieder von der Industrie gesponsort werden. Im Einzelnen sind dies:

\section{Redner- und Posterpreise}

2 Preise à 500 Euro für die besten Präsentationen im Rahmen der Jahrestagung. Die Preisträger für diese Preise werden im Rahme des Kongresses von einer Jury ausgewählt.

\section{Bauerfeind-Doktorandenpreis}

Der Doktorandenpreis richtet sich an junge Mediziner mit wissenschaftlichem Interesse auf den Gebieten Phlebologie, Lymphologie und verwandten Disziplinen. Bevorzugt werden Arbeiten, welche die Kompressionstherapie in die Untersuchungen einbeziehen. Vergeben wird ein Preisgeld in Höhe von 5000 Euro pro Preisträger (1500 Euro bei der Auszeichnung des Projekts und weitere 1500 Euro bei Vorlage der Dissertationsschrift - die verbleibenden 2000 Euro gehen an Erstbetreuer und Institution).

Bewerbungen und nähere Informationen erhalten Sie über medwiss@bauerfeind. com (z.Hd. Dr. A. Mark).

\section{Kreussler-Young- Phlebologists-Travel-Award}

Kreussler Pharma verleiht unter allen eingereichten Abstracts 2 Travel-Awards in Höhe von je 1000 Euro. Der Award wird regelmäßig im Rahmen dieses Kongresses zur Förderung des wissenschaftlichen Nachwuchses vergeben. Das mit jeweils 1000 Euro dotierte Reise-Stipendium soll Sie bei der Teilnahme am Kongress und der Präsentation Ihrer wissenschaftlichen Arbeit unterstützen.

\section{medi Reise- und Teilnahme- stipendien für Arzt-Berufs- starter}

medi unterstützt interessierte Ärztinnen und Ärzte, die am Anfang ihrer beruflichen Laufbahn stehen, mit Reise- und Teilnahmestipendien. Diese umfassen den freien Eintritt an allen Kongresstagen sowie die Fahrt- und Hotelkosten bis zu einem Betrag von 500 Euro. Alle Interessierten können sich mit einem Bewerbungsbogen und einer Einverständniserklärung seitens ihres Dienstherrn oder Arbeitgebers bewerben. Bewerbungsfrist ist der 28.6.2020.

Der Bewerbungsbogen und weitere Informationen können hier angefordert werden: medi, Frau Strauss, a.strauss@med.de.

\section{Sigvaris-Förderpreis Phlebologie}

Der Sigvaris-Förderpreis Phlebologie richtet sich an junge Wissenschaftler und Mediziner. Die Bewerber sollten Interesse an wissenschaftlicher Arbeit und Spaß an aktueller Berichterstattung haben. Die Verleihung erfolgt auf unserer Jahrestagung.

Sie können Ihre Bewerbung mit einem aussagefähigen Lebenslauf hier einreichen: cordula.berger@sigvaris.com.

\section{Juzo-Forschungspreis Phlebologie}

Der Juzo-Forschungspreis Phlebologie wird in Kooperation mit der Deutschen Gesellschaft für Phlebologie vergeben. Er soll die Wissenschaft in der interdisziplinären Kompressionstherapie unterstützen und wird vergeben an Mediziner mit wissenschaftlichem Interesse auf den Gebieten Phlebologie, Lymphologie, Dermatologie und verwandten Disziplinen. Gefördert werden Projektkonzepte, welche explizit die Kompressionstherapie in die Untersuchungen einbeziehen. Der Preis ist mit 5000 Euro ausgeschrieben und wurde im Rahmen der Jahrestagung der Deutschen Gesellschaft für Phlebologie erstmals 2019 in Münster vergeben. Bewerbungen richten Sie bitte bis zum 30.6.2020 an das Sekretariat der Deutschen Gesellschaft für Phlebologie: sekretariat@phlebology.de.

\section{Zoltan-Varady-Vortragspreis}

Herr Prof. Dr. Zoltan Varady stiftet in Kooperation mit der Deutschen Gesellschaft für Phlebologie einen Wissenschaftspreis im Rahmen unserer Jahrestagung. Jeweils der beste Vortrag im Bereich der Minichirurgie oder Mikrochirurgie wird mit einem Vortragspreis von 1000 Euro prämiert. Die Preisjury besteht aus 3 Mitgliedern der VOP-AG.

\section{IMPRESSUM}

Verantwortlich für Mitteilungen der DGP:

\section{Dr. med. Erika Mendoza}

